\title{
Wie Katz und Maus
}

\author{
Stefan Monecke, Christian Lück, Simone Trautmann, Matthias Meinhardt, \\ Enno Jacobs
}

Vorgeschichte und klinische Präsentation

Die Patientin M.S. ist eine 20-jährige Frau, bei der vor etwa 2 Jahren eine akute lymphoblastische Leukämie festgestellt wurde. Die Erstmanifestation war ein lymphozytäres Chlorom an der rechten Seite des Kopfes. Nach der operativen Entfernung des Chloromes erhielt Frau S. eine kurative Radiound Chemotherapie. Rund 18 Monate später kam es zu einem Rezidiv, das nach Konditionierung mit Cyclophosphamid, Anti-Thymozyten-Globulin und Ganzkörperbestrahlung mit einer allogenen Stammzelltransplantation behandelt wurde. Die Patientin war seit einem halben Jahr in einer kompletten Remission. Vor einigen Tagen bekam sie plötzlich Fieber bis zu $40^{\circ} \mathrm{C}$. Bis zur Aufnahme im Krankenhaus entwickelte sie Dyspnoe, Tachypnoe und etwas unproduktiven Husten.

Am Tag der Aufnahme hat die Patientin eine Leukozyten- bzw. Thrombozytenzahl von 3,0/nl und 108/nl. Das CRP ist mit $12 \mathrm{mg} / \mathrm{l}$ (Normwert $<5 \mathrm{mg} / \mathrm{l}$ ) leicht erhöht. Es werden mehrere Blutkulturen abgenommen, die aber alle steril bleiben. Ausserdem wird ein High-resolution-CT des Thorax durchgeführt, das aber keine signifikanten Veränderungen zeigt.

? Fragen

1. Welche Krankheitserreger spielen beim immunsupprimierten Patienten eine Rolle?

2. Welche Differenzialdiagnosen bestehen, welche Untersuchungen sollten daher angefordert werden?

3. Welche initiale antiinfektive Therapie würden Sie vorschlagen? 


\section{Weiterer klinischer Verlauf}

Die Patientin erhält Cotrimoxazol als Prophylaxe gegen Pneumocystis jiroveci (früher carinii). Außerdem werden »zur breiten Abdeckung aller möglichen Erreger« Imipenem, Fluconazol und Acyclovir gegeben. Der klinische Zustand verschlechtert sich innerhalb der folgenden Tage trotzdem, und die Patientin entwickelt eine disseminierte intravasale Gerinnung. Die Leukozytenzahl steigt auf 5,8/nl, das CRP steigt bis auf $329 \mathrm{mg} / \mathrm{l}$ und die LDH auf $2082 \mathrm{U} / \mathrm{l}$ (Normwert: 135-215 U/I). Die Thrombozyten fallen auf 69/ nl. Das Verhältnis $\mathrm{PaO}_{2} / \mathrm{FiO}_{2}$ als Ausdruck für die Schwere der Oxygenierungsstörung fällt bis auf $95 \mathrm{mmHg}$ (Normalwert: $350-450 \mathrm{mmHg}$ ). Deshalb wird die Patientin auf eine Internistische Intensivstation verlegt, intubiert und beatmet. Zusätzlich zu den genannten Antibiotika werden nun auch noch Erythromycin, Tobramycin und Vancomycin gegeben. Eine Röntgenaufnahme des Thorax zeigt ausgeprägte interstitielle Infiltrationen (• Abb. 43.1).

Für die mikrobiologische Diagnostik wird eine bronchoalveoläre Lavage (BAL) durchgeführt. Weder in den nach Gram noch den nach ZiehlNeelsen gefärbten Präparaten werden Krankheitserreger gesehen, und die bakteriologischen Kulturen bleiben negativ. Zwei verschiedene direkte Immunofluoreszenztests auf Pneumocystis jiroveci sind negativ. Zusätzlich wird ein Giemsa-Präparat angefertigt. In diesem Präparat wird eine hohe Zahl von »Halbmond-« oder »Mandarinenscheiben«-förmigen Organismen mit gut erkennbarem Zellkern entdeckt, die sowohl intra- als auch extrazellulär liegen (- Abb. 43.2.). Die Diagnose einer Toxoplasmagondii-Infektion wird durch einen positiven direkten Immunofluoreszenztest bestätigt. In einer Serumprobe vom gleichen Tag wird Toxoplasma IgM nachgewiesen. Eine Therapie mit Pyrimethamin, Sulfadizin und Clindamycin wird sofort begonnen, aber die Patientin stirbt am nächsten Tag an einem »adult respiratory distress syndrome« (ARDS).

Bei der Autopsie wird das Vorhandensein von Toxoplasma gondii, aber das Fehlen einer entzündlichen Reaktion in den Lungen, im Myokard und in den basalen Ganglien nachgewiesen.

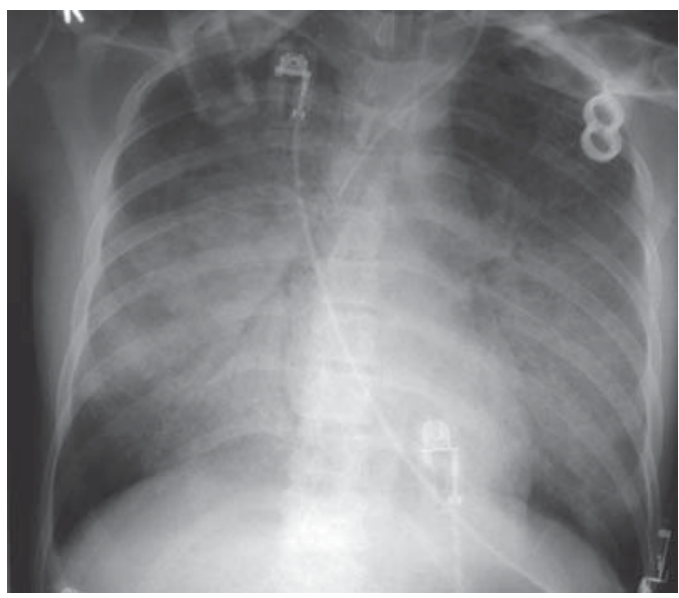

- Abb. 43.1. Thoraxröntgenaufnahme, die einen Tag vor dem Tod der Patientin aufgenommen wurde

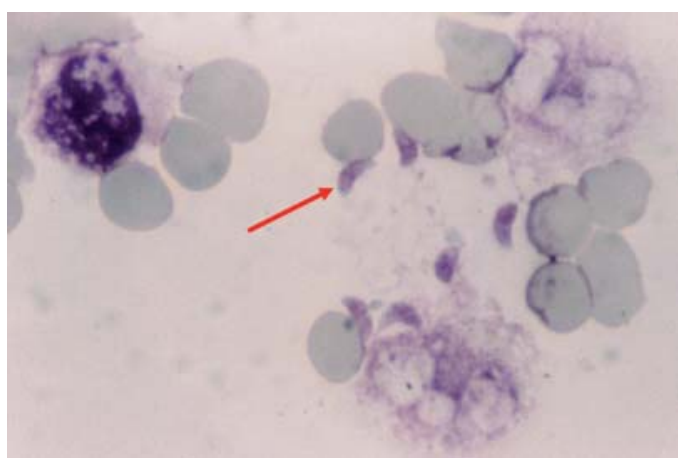

- Abb. 43.2. Giemsa-Präparat der BAL mit mehreren extrazellulären Trophozoiten von Toxoplasma gondii $(\times 1000)$

\section{Diagnose}

Disseminierte Toxoplasmose mit Primärmanifestation als ARDS

\section{Diskussion}

Toxoplasma gondii ist ein eukaryontischer Parasit, dessen Endwirt die Katze ist. Zahlreiche andere Tiere sind potentielle Zwischenwirte. Dazu gehören v.a. Mäuse, deren Verhalten durch den Parasiten so manipuliert wird, dass die Wahrscheinlichkeit, von einer Katze gefressen zu werden, steigt 
(Berdoy et al. 2000). Ob diese Manipulationen auch bei infizierten Menschen zu Verhaltensänderungen führen, ist ein interessantes Problem; eine neuere Studie hat bei Traumapatienten, die in Verkehrsunfälle verwickelt waren, im Vergleich zu Kontrollpatienten eine höhere Seroprävalenz nachweisen können (Flegr et al. 2002).

Menschen können infiziert werden, indem sie entweder von Katzen ausgeschiedene infektiöse Oozysten oder zystenhaltiges Fleisch anderer Zwischenwirte (v.a. Schweine, aber auch Rinder, Ziegen, Schafe und sogar Hühner) aufnehmen. Groß angelegten Untersuchungen in Mittel- und Westeuropa zufolge liegt die Durchseuchungsrate bei Frauen in gebärfähigem Alter bei $40-60 \%$. Normalerweise ist die Primärinfektion asymptomatisch oder uncharakteristisch mit geringgradigem Fieber und Lymphknotenschwellungen. Bei einer Erstinfektion während der Schwangerschaft kann es zur Infektion des Fetus kommen, die zu einem der Röteln- oder CMV-Embryopathie ähnlichen Syndrom führt und neurologische Defizite, Hydrocephalus und Taubheit verursachen kann. Deshalb sollten Schwangere engen Kontakt zu Katzen meiden und kein rohes Fleisch (Hackepeter bzw. Mett) essen.

Die Parasiten persistieren nach einer überstandenen Infektion im Menschen, wodurch es bei einer Immunsuppression $\mathrm{zu}$ einer Reaktivierung kommen kann. Die häufigste Form ist die Toxoplasmose des Zentralnervensystems, die zu typischen, abszessartigen Prozessen im Gehirn führt. Pulmonale und generalisierte Verlaufsformen sind seltener. Die Therapie erfolgt mit Pyrimethamin, Sulfadizin und Clindamycin. Die ersten beiden Substanzen wirken auf die Folsäuresynthese der Erreger. Clindamycin wirkt eigentlich nicht auf eukaryontische Ribosomen, sondern kommt dadurch zustande, dass es Organellen unbekannter Funktion (die Apicoplasten) abtötet, die phylogenetisch von freilebenden Prokaryonten abstammen. Spiramycin kann die Infektion des Fetus verhindern, ist aber für eine Therapie nicht wirksam genug.

Die hier beschriebene pulmonale Toxoplasmose ist eine sehr seltene Verlaufsform einer Toxoplasma-gondii-Reaktivierung bei Patienten mit Immunsuppression. Wegen der steigenden Zahl der Patienten, die Knochenmark- oder andere
Organtransplantationen erhalten, und wegen der HIV-Epidemie wurde dieses Krankheitsbild in den letzten Jahren auch in Deutschland zunehmend häufiger beobachtet.

Klinisch erscheint die pulmonale Toxoplasmose als schwere atypische Pneumonie. In einem 1996 publiziertem Review (Rabaud et al. 1996) wurden die Symptome von 64 Fällen ausgewertet. $70 \%$ der Patienten hatten Fieber über $39^{\circ} \mathrm{C}$ und $92 \%$ hatten Husten. Bei 6,2\% der Patienten entwickelte sich ein ARDS und bei 15,6\% ein septischer Schock. Die Mortalität betrug 37\%. Andere Studien geben Sterblichkeitsraten von 55\% (Pomeroy u. Filice 1992) bis über 90\% (Sing et al. 1999) an.

Bei immunsupprimierten Patienten mit sog. atypischer Pneumonie gibt es keine klinischen oder radiologischen Merkmale, die eine pulmonale Toxoplasmose von anderen atypischen Pneumonien (Pneumocystis-jiroveci-Pneumonie, Legionellose) unterscheiden können. Eine wichtige Differenzialdiagnose ist daher immer die Pneumocystis-jiroveci-Pneumonie (PCP). Sowohl bei der PCP als auch bei pulmonaler Toxoplasmose kommt es zu zunehmender Atemnot mit trockenem Husten und zur LDH-Erhöhung, wobei gerade der letztgenannte Laborwert bei einer Vielzahl anderer Erkrankungen ebenfalls deutlich erhöht sein kann. Bei beiden Pneumonieformen ist das Thoraxröntgenbild initial wenig auffällig, später treten unspezifische Veränderungen wie interstitielle Verschattungen auf.

Die mikrobiologische Diagnostik der beiden Erreger unterscheidet sich methodisch erheblich: Während zum Nachweis von Pneumocystis jiroveci kommerziell erhältliche direkte Immunofluoreszenztests sowie teilweise sog. In-house-PCR-Tests Verwendung finden, wird die Diagnose einer Toxoplasmose beim Immunkompetenten meist serologisch gestellt. Beim Immunsupprimierten können damit allerdings nicht regelhaft zuverlässige Befunde erhoben werden. In Einzelfällen können serologische Befunde (Zunahme der IgG-Titer oder positives IgM) zur Diagnose einer pulmonalen Toxoplasmose beitragen. Rabaud (Rabaud et al. 1996) berichtete über 64 Patienten mit pulmonaler oder generalisierter Toxoplasmose, von denen 3 Patienten serokonvertierten, 12 einen mehr als 
2-fachen Anstieg des IgG-Titers und einer ein erhöhtes IgM hatten.

Beim Immunsupprimierten ist ein direkter Erregernachweis anzustreben. Da die PCR-Untersuchung noch kein standardisiertes Verfahren darstellt, ist die Giemsa-Färbung die Diagnostik der Wahl: Trophozoiten von Toxoplasma gondii erscheinen als charakteristisch geformte Gebilde (to$x o n=$ Bogen, plasma $=$ Form) von ungefähr $6 \times 2 \mu \mathrm{m}$, die rote Kerne enthalten (• Abb.43.2). Sie treten sowohl intra- als auch extrazellulär auf (Bottone 1991). Die Wahrscheinlichkeit, Toxoplasmen zu finden, hängt sowohl von der Zahl der Organismen in der Probe als auch von der Geduld und Erfahrung des Beobachters ab. In der Studie von Sing (Sing et al. 1999) wurden in 7 von 8 gesicherten Fällen Toxoplasmen in der BAL gesehen. Die gleiche Arbeit zeigt auch, daß die Giemsa-Färbung mindestens genauso empfindlich ist wie die direkte Immunofluoreszenz. In dem Review von Rabaud (Rabaud et al. 1996) erbrachte eine Kombination beider Methoden bei 57 von 64 Patienten (89\%) positive Resultate.

Der Nachweis von Toxoplasma gondii in Versuchstieren oder Zellkulturen ist auch möglich, aber nur wenige Labors haben die Möglichkeiten, solche Versuche durchzuführen.

Wichtig

Bei Patienten mit Immunsuppression, insbesondere nach Knochenmarktransplantation, ist der fulminante Verlauf von Systeminfektionen mit Toxoplasma häufig nicht vorhersehbar, wobei frühzeitig ein direkter Erregernachweis aus geeigneten Materialien anzustreben ist.

\section{Literatur}

Berdoy M, Webster JP et al. (2000) Fatal attraction in rats infected with Toxoplasma gondii. Proc R Soc Lond B Biol Sci 267:1591-1594

Bottone EJ (1991) Diagnosis of acute pulmonary toxoplasmosis by visualization of invasive and intracellular tachyzoites in Giemsa-stained smears of bronchoalveolar lavage fluid. J Clin Microbiol 29:2626-2627

Flegr J, Havlicek J et al. (2002) Increased risk of traffic accidents in subjects with latent toxoplasmosis: a retrospective case-control study. BMC Infect Dis 2:11

Pomeroy C, Filice GA (1992) Pulmonary toxoplasmosis: a review. Clin Infect Dis 14: 863-870
Rabaud C, May T et al. (1996) Pulmonary toxoplasmosis in patients infected with human immunodeficiency virus: a French National Survey. Clin Infect Dis 23:1249-1254

Sing A, Leitritz L et al. (1999) Pulmonary toxoplasmosis in bone marrow transplant recipients: report of two cases and review. Clin Infect Dis 29:429-433 